10. Rao, A. M. et al. Diameter-selective Raman scattering from vibrational modes in carbon nanotubes. Science 275, 187-191 (1997)

11. Kane, C. L. \& Mele, E. J. Size, shape, and low-energy electronic structure of carbon nanotubes. Phys. Rev. Lett. 78, 1932-1935 (1997).

12. Haddon, R. C. et al. Conducting films of $\mathrm{C}_{60}$ and $\mathrm{C}_{70}$ by alkali metal doping. Nature 350, 320-232 (1991).

13. Rao, A. M., Eklund, P. C., Bandow, S., Thess, A. \& Smalley, R. E. Evidence for charge transfer in doped carbon nanotube bundles from Raman scattering. Nature 388, 257-259 (1997).

14. Dahn, J. R., Zheng, T., Liu, Y. H. \& Xue, J. S. Mechanisms for lithium insertion in carbonaceous materials. Science 270, 590-593 (1995).

15. Balents, L. \& Fisher, M. P. A. Correlation effects in carbon nanotubes. Phys. Rev. Lett. 55, 11973-11976 (1997).

16. Mintmire, J. W., Robertson, D. H. \& White, C. T. Properties of fullerene nanotubes. J. Phys. Chem. Solids 54, 1835-1840 (1993).

Acknowledgements. H.J.K. is on leave from Hallym University, South Korea. We thank M. Clement for experimental assistance. Work at Penn was supported by the Department of Energy; work at Rice was supported by the Office of Naval Research and the Robert A. Welch Foundation.

Correspondence and requests for materials should be addressed to J.E.F. (e-mail: fischer@soll.lrsm. upenn.edu)

Evidence for charge transfer in doped carbon nanotube bundles from Raman scattering

A. M. Rao ${ }^{\star}$, P. C. Eklund ${ }^{\star}$, Shunji Bandow $\dagger$, A. Thess $\ddagger$ \& R. E. Smalley $\ddagger$

${ }^{*}$ Department of Physics and Astronomy and Center for Applied Energy Research, University of Kentucky, Lexington, Kentucky 40506-0055, USA

$\dagger$ Instrument Center, Institute for Molecular Science, Myodaiji, Okazaki, 444, Japan

$\ddagger$ Center for Nanoscale Science and Technology, Rice Quantum Institute and Departments of Chemistry and Physics, Rice University, Houston, Texas 77251, USA

Single-walled carbon nanotubes ${ }^{1}$ (SWNTs) are predicted to be metallic for certain diameters and pitches of the twisted graphene ribbons that make up their walls ${ }^{2}$. Chemical doping is expected to substantially increase the density of free charge carriers and thereby enhance the electrical (and thermal) conductivity. Here we use Raman spectroscopy to study the effects of exposing SWNT bundles $^{1}$ to typical electron-donor (potassium, rubidium) and electron-acceptor (iodine, bromine) dopants. We find that the high-frequency tangential vibrational modes of the carbon atoms in the SWNTs shift substantially to lower (for $K, R b$ ) or higher (for $\mathrm{Br}_{2}$ ) frequencies. Little change is seen for $\mathrm{I}_{2}$ doping. These shifts provide evidence for charge transfer between the dopants and the nanotubes, indicating an ionic character of the doped samples. This, together with conductivity measurements ${ }^{3}$, suggests that doping does increase the carrier concentration of the SWNT bundles.

We generate SWNT bundles by a dual pulsed-laser technique ${ }^{1}$, which is known to produce a very narrow diameter distribution peaked around the diameter of the $(10,10)$ ('armchair') nanotube (here the indices refer to the diameter and pitch of the tubes). The nanotubes are grouped into aligned bundles of as many as 100-500 tubes, packed into a triangular lattice. The chemical doping of the SWNT bundles was carried out in sealed quartz ampoules using vapour-distilled reactants. Our method parallels those used to intercalate graphite ${ }^{4}$. In the SWNT bundles studied here, the dopant is not expected to lie inside the tube; it may be confined to the interstitial channels found between the tubes in the bundles, or may decorate the entire exterior surface of the SWNTs. If the tubule ends were open, however, it should also be possible to place dopant ions or molecules inside the tubes, as has been done with very small $(\sim 1.2 \mathrm{~nm}$ inside diameter $)$ multi-wall nanotubes ${ }^{5,6}$.
The reactant and SWNT sample were positioned at opposite ends of an evacuated ampoule; the reactant vapour travels to the far end of the ampoule where the reaction takes place. The reaction with $\mathrm{I}_{2}$ and $\mathrm{Br}_{2}$ were carried out with both the sample and reactant at room temperature; the reaction is quite fast with $\mathrm{Br}_{2}$ (minutes), whereas $\mathrm{I}_{2}$ requires several hours to saturate the sample. The alkali-metal reactions were both carried out with the alkali metal and SWNTs at elevated temperatures for $24 \mathrm{~h}$ (metal temperature, $120^{\circ} \mathrm{C}$; SWNT temperature, $160^{\circ} \mathrm{C}$ ). Raman scattering spectra were then measured while the samples remained sealed in their reaction ampoules. All the spectra reported here were measured in the backscattering configuration using 514.5-nm laser excitation. The scattered light was analysed in a Jobin Yvon HR460 single-grating spectrometer equipped with a charge-coupled array detector and a holographic notch filter (Kaiser Optical Systems, Inc., Ann Arbor, MI, USA). To avoid laser damage to the sample, all the data were taken at low laser power $\left(2 \mathrm{~W} \mathrm{~cm}^{-2}\right)$ using a cylindrical lens to focus the laser radiation onto the sample (which was a loosely compacted mat of SWNTs). No polarization analyser was used, so that light polarized both perpendicular and parallel to the scattering plane was collected. Figure 1 shows the $T=300 \mathrm{~K}$ Raman spectra of pristine SWNTs and those doped with the anticipated electronacceptor $\left(\mathrm{I}_{2}, \mathrm{Br}_{2}\right)$ and electron-donor $(\mathrm{K}, \mathrm{Rb})$ reagents.

We first discuss briefly the Raman spectrum for pristine SWNTs (Fig. 1, middle spectrum). As discussed previously ${ }^{7}$, the strong peak at $186 \mathrm{~cm}^{-1}$ has been identified with the $A_{g}$ symmetry radial breathing mode, and the peak at $1,593 \mathrm{~cm}^{-1}$ has been assigned to an unresolved Raman triplet identified with tangential $\mathrm{C}$-atom displacement modes, one of $\mathrm{A}_{g}$ and two with $\mathrm{E}_{g}$ symmetry. These three, nearly degenerate, tubule phonons are related to phonons in the highest-frequency optical phonon branch of graphite which exhibits a zone centre $\mathrm{E}_{2 g}$ symmetry intralayer mode at $1,582 \mathrm{~cm}^{-1}$. The relative peak intensities of all the Raman-active modes depend critically on the laser wavelength which indicates that the scattering is resonant ${ }^{7}$. For the assignments of the weaker peaks in the pristine spectrum, see ref. 7 .

We next address the changes in the Raman spectrum of the pristine tubes due to chemical doping (Fig. 1). The top two spectra in Fig. 1 are for SWNT bundles reacted with typical electronacceptor dopants $\left(\mathrm{I}_{2}, \mathrm{Br}_{2}\right)$ which are expected to transfer electrons from the carbon $\pi$ states in the tubules to the dopant molecules, creating hole carriers in the SWNTs. The $\mathrm{I}_{2}$-doping induces the smallest change in the SWNT Raman spectrum. This is perhaps not surprising, as $\mathrm{I}_{2}$ does not intercalate into graphite. It does diffuse into solid $\mathrm{C}_{60}$ (refs 8,9$)$, but no convincing evidence for charge transfer with the fullerene lattice has been reported. For the chemically doped SWNTs, we assign the most intense low-frequency and high-frequency modes to the radial and tangential modes, respectively. Iodine-doping of SWNT bundles is seen to slightly increase the radial mode frequency from 186 to $188 \mathrm{~cm}^{-1}$ $\left(\Delta_{\mathrm{r}}=+2 \mathrm{~cm}^{-1}\right)$, and slightly decrease the tangential mode frequencies from $1,593 \mathrm{~cm}^{-1}$ in the pristine SWNT to $1,590 \mathrm{~cm}^{-1}$ $\left(\Delta_{\mathrm{t}}=-3 \mathrm{~cm}^{-1}\right)$, where $\Delta_{\mathrm{r}}$ and $\Delta_{\mathrm{t}}$ refer to the change in the radial and tangential mode frequencies on doping. For the case of saturated $\mathrm{Br}_{2}$-doping, relatively large increases in the radial and tangential mode frequencies are observed: $\Delta_{\mathrm{r}}=+74 \mathrm{~cm}^{-1}$ and $\Delta_{\mathrm{t}}=+24 \mathrm{~cm}^{-1}$. We note that the $1,617 \mathrm{~cm}^{-1}$ peak in the $\mathrm{Br}_{2^{-}}$ doped SWNT decreases in frequency to $1,603 \mathrm{~cm}^{-1}$ on refreezing the bromine vapour at the opposite end of the reaction tube; that is, the peak does not shift all the way back to the pristine value at $1,593 \mathrm{~cm}^{-1}$. This suggests the existence of an intermediate-concentration $\mathrm{Br}_{2}$-doped phase, in agreement with the resistivity studies on $\mathrm{Br}_{2}$-doped SWNT ${ }^{3}$. Also in agreement with these resistivity studies ${ }^{3}$, the Raman spectrum of saturated $\mathrm{Br}_{2}$-doped SWNT bundles shown in Fig. 1 is recovered again when the $\mathrm{Br}_{2}$ returns to room temperature and the increased vapour pressure drives the $\mathrm{Br}_{2}-\mathrm{SWNT}$ system back to the $\mathrm{Br}_{2}$-saturated phase. 


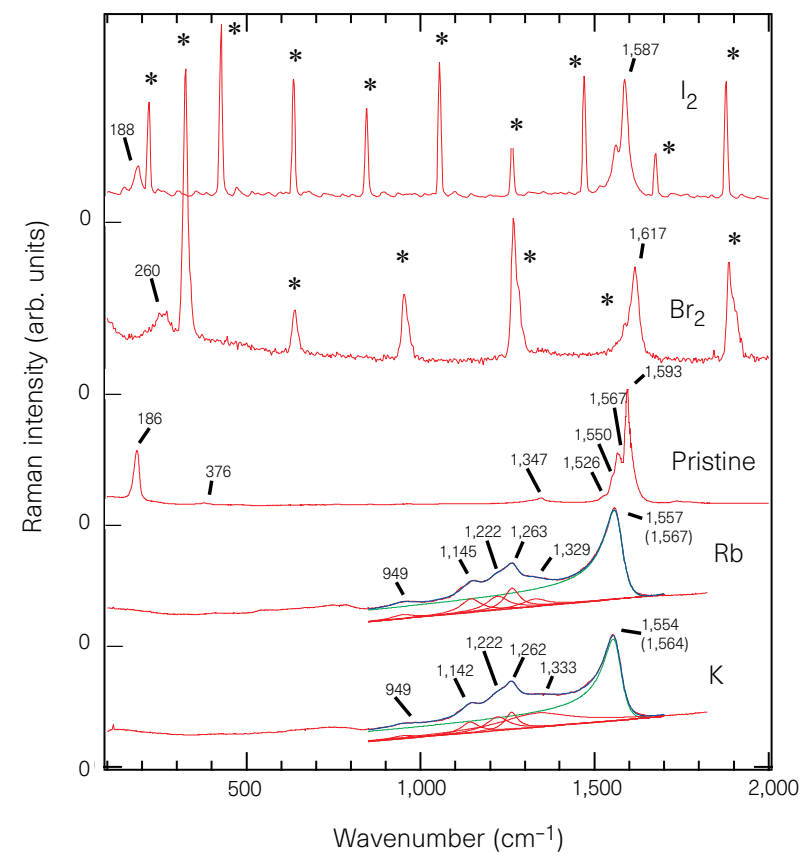

Figure 1 Raman scattering spectra for pristine SWNT bundles reacted with various donor and acceptor reagents. From top to bottom: $\mathrm{I}_{2}, \mathrm{Br}_{2}$, pristine SWNT, $\mathrm{Rb}$ and $\mathrm{K}$. The backscattered spectra were taken at $T=300 \mathrm{~K}$ using 514.5-nm radiation. In the spectra for both of the halogen-doped SWNT bundles, a harmonic series of peaks (indicated with an asterisk) is observed, which are identified with the fundamental stretching frequency $\omega_{\mathrm{s}}$ : $\sim 220 \mathrm{~cm}^{-1}\left(\mathrm{I}_{2}\right)$ and $\sim 324 \mathrm{~cm}^{-1}\left(\mathrm{Br}_{2}\right)$. The spectra have been scaled so that the strongest SWNT feature appears to have the same intensity. In the vicinity of the strongest highfrequency mode around $1,550 \mathrm{~cm}^{-1}$, the Raman spectra for SWNTs doped with Kor $\mathrm{Rb}$ are fitted with a superposition of lorentzian functions (red curves) and an asymmetric Breit-Wigner-Fano lineshape (green curve) on a linear continuum; see text. In both cases, the calculated spectrum (blue curve) is nearly superimposed on the data. Peak frequencies are indicated; the values in parentheses are the renormalized phonon frequencies (see text).

The two spectra at the bottom of Fig. 1 are for SWNTs that have reacted with typical electron-donor reagents $(\mathrm{K}, \mathrm{Rb})$ which transfer electrons to itinerant carbon $\pi^{\star}$ states in the SWNTs. As can be seen in the figure, the low-frequency radial breathing mode is not evident in the spectrum. It may have shifted below our lowest detectable frequency $\left(\sim 100 \mathrm{~cm}^{-1}\right)$, or, alternatively, it may have broadened to such an extent that it cannot be detected above the background. A noticeably large, and nearly identical, downshift of all the highfrequency Raman structure is observed for both the $\mathrm{K}$ and $\mathrm{Rb}$ dopants, even though the $\mathrm{Rb}$ atom has a much larger diameter and mass than the $\mathrm{K}$ atom. The broad, asymmetric peak at $\sim 1,565 \mathrm{~cm}^{-1}$ observed in both alkali-metal-doped SWNT bundles is tentatively identified with a Breit-Wigner-Fano (BWF) interference ${ }^{10}$. In solids, this resonance usually involves an interference between Raman scattering from continuum excitations and that from a discrete phonon, provided the two Raman-active excitations are coupled. The degree of coupling $(1 / q)$ determines the departure of the lineshape from a symmetric lorentzian function. Similar broad BWF resonances are observed in stage 1 donor graphite intercalation compounds (GICs; for example, $\mathrm{MC}_{8}$, where $\mathrm{M}$ is $\mathrm{K}, \mathrm{Rb}, \mathrm{Cs}$ ), and not in the lower charge transfer, stage 2 donor GICs (for example, $\mathrm{MC}_{24}$, where $\mathrm{M}$ is $\mathrm{K}, \mathrm{Rb}, \mathrm{Cs}$ ). The positions of the BWF peaks in the stage 1 GICs are slightly lower than those observed here. We assign the BWF peak in the alkali-metal-doped SWNTs to interference scattering between either an electronic or multiphonon continuum and the highest-frequency tube phonons which have been softened by electron transfer.

When the coupling between the discrete mode and the continuum is significant, the BWF peak position can be considerably displaced from the frequency of the uncoupled discrete phonon ${ }^{10}$. This is referred to as a renormalization of the 'bare' phonon frequency. If the continuum scattering in the SWNTs is electronic in origin, then the degree of frequency renormalization can be an important measure of the electron-phonon interaction. This aspect of the BWF resonance will be addressed elsewhere. The BWF lineshape is given by

$$
I(\omega)=I_{0}\left\{1+\left(\omega-\omega_{0}\right) / q \Gamma\right\}^{2} /\left\{1+\left(\left(\omega-\omega_{0}\right) / \Gamma\right)^{2}\right\}
$$

where $I_{0}, \omega_{0}$ and $\Gamma$ are, respectively, the intensity, renormalized frequency and broadening parameter. In Fig. 1, we show a fit (blue curve) to the high-frequency experimental spectra for Rb- and KSWNTs. The solid red curves represent the individual lorentzian peak shapes used for the peaks at $\sim 949,1,145,1,222,1,263$ and $1,329 \mathrm{~cm}^{-1}$ and the BWF lineshape (green curve) with its peak at $1,567 \mathrm{~cm}^{-1}$. The lorentzian peaks in the figure are tentatively assigned to charge-transfer-softened modes; they are not observed in the pristine SWNT spectrum. The doping-induced increase in the Raman-activity of these mid-frequency phonons is not understood; it may be related to a breakdown in the pristine nanotube selection rules associated with the doping. The linewidths are also very large, suggesting broadening through either the electron-phonon interactions or inhomogeneous doping. Assignment of these midfrequency $\left(\sim 900-1,200 \mathrm{~cm}^{-1}\right)$ features must await further work.

BWF lineshapes have been observed previously in doped $\mathrm{M}_{3} \mathrm{C}_{60}$ (where $\mathrm{M}$ is $\mathrm{K}, \mathrm{Rb})^{11}$ and $\mathrm{MC}_{8}$ (where $\mathrm{M}$ is $\mathrm{K}, \mathrm{Rb}, \mathrm{Cs}$ ) GICs ${ }^{4}$. The negative value of $1 / q=-0.35$ we observe for the alkali-metaldoped SWNT bundles is about a factor of three smaller than those observed for the broad BWF resonance in $\mathrm{MC}_{8}$ compounds ${ }^{12}$. Similar values of $1 / q$ to those found here have been reported for the low-frequency $\mathrm{H}_{g}$ symmetry, intramolecular vibrations in $\mathrm{M}_{3} \mathrm{C}_{60}$ (ref. 11).

We now consider if the doping-induced shifts in the SWNT phonon frequencies that we observe are consistent with the results of the Raman studies of GICs (ref. 4), and weight uptake measurements on doped SWNTs by Lee et al. in the companion Letter ${ }^{3}$. We appeal to results of previous studies of charge-transfer-induced changes in the high-frequency intralayer mode $\left(\mathrm{E}_{2 g 2}\right)$ in acceptor and donor GICs (for a review, see ref. 4). In these compounds, charge is exchanged between the intercalated layer and the adjacent (or 'bounding') carbon layers. The degree of charge transfer may be defined by the quantity $f$ which is the transferred charge per host $\mathrm{C}$ atom. For example, in $\mathrm{KC}_{8}$, the charge transfer is essentially complete (the $\mathrm{K}$ is almost fully ionized to $\mathrm{K}^{+}$) and $f \approx 1 / 8$ (ref. 12). Using stage 1 and stage 2 Raman data from alkali-metal-doped $\mathrm{GICs}^{4}$, we estimate the average value $\Delta \omega / \Delta f \approx-140 \mathrm{~cm}^{-1}$, where the minus sign indicates that the intralayer mode softens with increasing charge transfer into the $\pi^{\star}$ band. For the acceptor compounds, we use results from Raman scattering studies of the continuous electrochemical charging of the $\mathrm{C}$ layers in stage 1 graphite- $\mathrm{H}_{2} \mathrm{SO}_{4}$ (ref. 13) from which $\Delta \omega / \Delta f \approx+460 \mathrm{~cm}^{-1}$ is obtained; that is, the intralayer mode stiffens with the introduction of holes into the $\pi$ band.

For $\mathrm{Br}_{2}$, which is known to be a weak acceptor (that is, it has a small $f$ value) in a GIC, and using $\Delta \omega / \Delta f \approx+460 \mathrm{~cm}^{-1}$, we estimate $f \approx 1 / 19$, or one free hole per $19 \mathrm{C}$ atoms in the SWNT. However, weight uptake measurements by Lee et al. ${ }^{3}$ indicate that an effective composition $\mathrm{C}_{52} \mathrm{Br}_{2}$ has been obtained under similar reaction conditions. Under these assumptions, we must therefore conclude that the acceptor SWNTs exhibit a much larger value of $\Delta \omega / \Delta f$ than observed in GICs. For $\mathrm{I}_{2}$-doped SWNTs, the shift of the $1,593 \mathrm{~cm}^{-1}$ peak is quite small, consistent with either very weak charge transfer between $\mathrm{I}_{2}$ and the tube wall or simply a physisorption of dopant on the tube wall. In fact, the frequency decreases slightly, as if the $I_{2}$ 
behaves as a donor, which is not expected.

For the alkali-metal dopants $\mathrm{K}$ and $\mathrm{Rb}$, the high-frequency modes show a BWF interference lineshape. As discussed above, this interference introduces a frequency renormalization for the high-frequency modes. Without a detailed analysis involving the spectral shape of the interfering continuum excitations, as we performed earlier for $\mathrm{CsC}_{8}$ (ref. 10), we cannot separate the two contributions (that is, charge transfer and interference coupling $(1 / q)$ ) to the phonon frequency $\omega_{0}$ (equation (1)). Consequently, a quantitative statement about the charge-transfer-induced shift in the alkalimetal-doped SWNTs cannot be made at present. Although the onedimensional nature of the SWNTs is expected to alter the nature of the continuum excitations, the $T \approx 300 \mathrm{~K}$ BWF frequency $\omega_{0}$ of $\sim 1,567 \mathrm{~cm}^{-1}(\mathrm{Rb})$ and $1,565 \mathrm{~cm}^{-1}(\mathrm{~K})$ observed in the doped SWNT bundles is remarkably close to that in the stage $1 \mathrm{MC}_{8}$ GICs: $1,547 \mathrm{~cm}^{-1}\left(\mathrm{KC}_{8}\right)$ and $1,519 \mathrm{~cm}^{-1}\left(\mathrm{CsC}_{8}\right)$ for $T \approx 300 \mathrm{~K}$. The coupling constants $(1 / q)$ are, however, a factor of $\sim 3$ higher in the GICs. We can say that the alkali-metal-doped SWNT Raman spectra qualitatively indicate that the bundles are highly doped, which is consistent with the large weight uptake (effective composition $\mathrm{KC}_{8}$ ) observed by Lee et al. ${ }^{3}$.

We have also investigated the temperature stability in vacuum of the alkali-metal-doped SWNT bundles. A series of Raman spectra were taken on a Rb-doped SWNT sample as a function of temperature in the range $24-380^{\circ} \mathrm{C}$. The spectra were measured with the $\mathrm{Rb}$-containing end of the evacuated ampoule at room temperature while the sample end of the ampoule was heated to progressively higher temperatures and equilibrated. A frequency decrease, probably associated with the thermal expansion of the SWNTs, and a broadening of the BWF peak, was observed at the highest temperatures. Interestingly, near $\sim 200^{\circ} \mathrm{C}$, sharp Raman peaks around 1,480 and $1,590 \mathrm{~cm}^{-1}$ were observed as shoulders on the BWF resonance, suggesting the existence of a metastable phase near $200{ }^{\circ} \mathrm{C}$ with a different structural arrangement for the $\mathrm{Rb}$ ions. At elevated temperatures, these additional peaks disappeared. At the highest temperature $\left(380^{\circ} \mathrm{C}\right)$, the Raman spectra suggests that the $\mathrm{Rb}$ remains bonded to the tube wall. In situ studies of $\mathrm{Br}_{2}$ uptake at $T \approx 300 \mathrm{~K}$ in these SWNT bundles also reveal an intermediateconcentration phase. In this case, the high-frequency peak at $1,593 \mathrm{~cm}^{-1}$ was found to quickly $(<5 \mathrm{~min})$ increase in frequency on exposure to bromine vapour and attain an intermediate value $\left(1,603 \mathrm{~cm}^{-1}\right)$; after some time, the frequency changed rapidly to the saturation-doped value $\left(1,617 \mathrm{~cm}^{-1}\right)$.

Received 21 February; accepted 3 June 1997

1. Thess, A. et al. Crystalline ropes of metallic carbon nanotubes. Science 273, 483-487 (1996).

2. Dresselhaus, M. S., Dresselhaus, G. \& Eklund, P. C. Science of Fullerenes and Carbon Nanotubes Ch. 19 (Academic, San Diego, 1996).

3. Lee, R. S., Kim, H. J., Fischer, J. E., Thess, A. \& Smalley, R. E. Conductivity enhancement in singlewalled carbon nanotube bundles doped with K and Br. Nature 388, 255-257 (1997).

4. Dresselhaus, M. S. \& Dresselhaus, G. Intercalation compounds of graphite. Adv. Phys. 30, 139-326 (1981).

5. Ajayan, P. M. \& Iijima, S. Capillarity-induced filling of carbon nanotubes. Nature 361, 333-334 (1993).

6. Ajayan, P. M. et al. Opening carbon nanotubes with oxygen and implications for filling. Nature 362 522-525 (1993)

7. Rao, A. M. et al. Diameter-selective Raman scattering from vibrational modes in carbon nanotubes. Science 275, 187-191 (1997)

8. Zhu, Q. et al. Intercalation of solid $\mathrm{C}_{60}$ with iodine. Nature 355, 712-714 (1992).

9. Kobayashi, M. et al. X-ray diffraction study of iodine-doped $\mathrm{C}_{60}$. Solid State Commun. 81, 93-95 (1992).

10. Eklund, P. C. \& Subbaswamy, K. R. Analysis of Breit-Wigner line shapes in the Raman spectra of graphite intercalation compounds. Phys. Rev. B 20, 5157-5161 (1979).

11. Zhou, P., Wang, K. A., Eklund, P. C., Dresselhaus, M. S. \& Dresselhaus, G. Raman scattering study of the electron-phonon interaction in $\mathrm{M}_{3} \mathrm{C}_{60}$. Phys. Rev. B 48, 8412-8417 (1993).

12. Eklund, P. C., Dresselhaus, G., Dresselhaus, M. S. \& Fischer, J. E. Raman scattering from in-plane modes in low-stage graphite-alkali-metal compounds. Phys. Rev. B 16, 3330-3333 (1977).

13. Eklund, P. C., Arakawa, E. T., Zarestky, J. L., Kamitakahara, W. A. \& Mahan, G. D. Charge-transferinduced changes in the electronic and lattice vibrational properties of acceptor-type GICs. Synth. Metals 12, 97-102 (1985).

Acknowledgements. We thank L. Grigorian (Univ. Kentucky) for experimental assistance. We also thank K. R. Subbaswamy and E. Richter (Univ. Kentucky), and M. S. Dresselhaus and G. Dresselhaus (MIT), for discussions. This work was supported by the NSF and DDE.

Correspondence and requests for materials should be addressed to P.C.E. (e-mail: eklund@pop.uky.edu).

\section{Seismological evidence for three-dimensional melt migration beneath the East Pacific Rise}

\section{Robert A. Dunn \& Douglas R. Toomey}

Department of Geological Sciences, University of Oregon, Eugene, Oregon 97403, USA

The extent to which crustal processes along mid-ocean ridges are controlled by either the pattern of mantle upwelling or the mode of magma injection into the crust is not known. Models of mantle upwelling vary from two-dimensional, passive flow ${ }^{1}$ to threedimensional, diapiric flow ${ }^{2-4}$. Similarly, beneath a ridge segment bounded by tectonic offsets, crustal magma chambers may be replenished continuously along the ridge $\mathrm{e}^{5-7}$ or at a central injection $z^{2} e^{2-4}$ from which magma migrates towards the segment's ends. Here we present tomographic images that reveal the seismic structure and anisotropy of the uppermost mantle beneath the East Pacific Rise. The anisotropy is consistent with two-dimensional mantle flow diverging from the rise, whereas the anomalous isotropic structure requires a three-dimensional but continuous distribution of melt near the crust-mantle interface. Our results indicate that crustal magma chambers are replenished at closely spaced intervals along-axis and that crustal systems inherit characteristics of scale from melt transport processes originating in the mantle.

The site of our investigation lies midway along a $100-\mathrm{km}-\mathrm{long}$ section of the East Pacific Rise (EPR) bounded by the $9^{\circ} 03^{\prime} \mathrm{N}$ overlapping spreading centre and the Clipperton transform, a comparatively well studied section of oceanic ridge (Fig. 1). Its broad axial summit ${ }^{2}$, axial-magma-chamber (AMC) reflector ${ }^{8,9}$, crustal low seismic-wave velocity ${ }^{6,10}$, and low- $Q$ zone ${ }^{11}$ are characteristic of a fast-spreading ridge that is magmatically robust. Apart from the overlapping spreading centre, the rise axis is continuous except for a series of fine-scale morphological discontinuities where the local trend of the rise changes abruptly by $10^{\circ}-15^{\circ}($ refs $2,5,12)$. Such deviations from axial linearity ('devals') segment the rise at intervals of $10-20 \mathrm{~km}$ and show no off-axis trace ${ }^{2,5}$. Devals are thought to demarcate axial hydrothermal systems ${ }^{12}$, petrologically distinct lava ${ }^{5,13}$ and the axial continuity of the AMC reflector'. Crustal tomography ${ }^{6,11,14}$ reveals an axially varying seismic structure that also correlates with the segmentation observed in sea-floor morphology and geology. As comparatively little is known about the structure at sub-crustal depths, the origin of this segmentation is enigmatic. It may derive from magmatic processes occurring in the mantle or from tectonic and hydrothermal processes confined to the crustal carapace.

Our tomographic analysis includes delay times from 1,362 crustal and $200 \mathrm{P}_{\mathrm{n}}$ (mantle) refractions (Fig. 2a). Examination of Fig. 2b, which shows delay times for sets of receivers on opposing sides of the rise, suggests that the data are consistent with both seismic heterogeneity and anisotropy within the uppermost mantle. To permit imaging in anisotropic media, we modified our tomographic method $^{14}$ to allow an azimuthally anisotropic upper mantle, a symmetry system in accord with previous studies of oceanic $P_{n}$ arrivals (see review in ref. 15). The velocity of $P_{n}$ waves for this symmetry system follows a $\cos (2 \theta)$ relationship, where $\theta$ is the azimuth of energy propagation ${ }^{15}$. We define a slowness model (slowness is the inverse of velocity) as

$$
\mathrm{u}^{\prime}\left(\mathrm{r}, \theta_{\mathrm{P}} ; \mathrm{u}(\mathrm{r}), \mathrm{a}(\mathrm{r}), \theta(\mathrm{r})\right)=\frac{\mathrm{u}(\mathrm{r})}{1+\frac{\mathrm{a}(\mathrm{r})}{2} \cos \left\lceil 2\left(\theta(\mathrm{r})-\theta_{\mathrm{P}}\right)\right\rceil}
$$

